TECHNICAL NOTE

\author{
J. Cousins \\ V. Haughton
}

\section{Motion of the Cerebellar Tonsils in the Foramen Magnum during the Cardiac Cycle}

\begin{abstract}
SUMMARY: Tonsil motion was measured with cine MR imaging in patients clinically suspected of having Chiari I malformation. Cardiac-gated sagittal 2D fast imaging employing steady-state acquisition (2D FIESTA) was performed in 11 patients with a Chiari I malformation and in 6 subjects without tonsil ectopia. Tonsil motion was measured through the cardiac cycle with a pixel-shift program designed for that purpose. Tonsil displacements in patients with Chiari I and those with normal cerebellar tonsils were compared. In patients with Chiari I and subjects with normal cerebellar tonsils, 2D FIESTA showed small-amplitude tonsil movement in cephalad and caudad directions during the cardiac cycle. The average total magnitude of motion was $0.43 \mathrm{~mm}$ in subjects with normal tonsils (controls) and 0.57 $\mathrm{mm}$ in patients with Chiari malformations, $33 \%$ greater than that in controls. Tonsil motion was 0.61 $\mathrm{mm}$ in the patients with syringomyelia and $0.50 \mathrm{~mm}$ in those without it (22\% difference).
\end{abstract}

$\mathbf{C}^{\mathrm{h}}$ haracterizing cerebellar tonsil motion during the cardiac cycle is important because its motion may contribute to the formation of syringomyelia in the Chiari I malformation. ${ }^{1}$ This study was designed to measure cyclic motion of the tonsils in the foramen magnum during the cardiac cycle with a cine MR imaging method, 2D fast imaging employing steadystate acquisition (2D FIESTA). The hypotheses tested were that tonsil motion is greater in patients with Chiari I than in controls and that tonsil motion is greater when syringomyelia is present.

Previous studies have reported divergent results for tonsil movement. For example, in patients undergoing cranio-occipital decompression, sonography has shown rapid tonsil motion. ${ }^{1}$ However, cardiac-gated phase-contrast MR (PC MR) imaging measurements suggested that the tonsils move minimally in healthy subjects during the cardiac cycle. ${ }^{2-5}$ Tonsil motion has also been measured with M-mode MR imaging and with echoplanar imaging. ${ }^{6,7}$ The motion of the cerebellar tonsils in patients with Chiari I has not, to our knowledge, been studied with cine methods such as those used in this report.

\section{Methods}

This study was performed with the approval of the local institutional review board, which granted a waiver of consent for the retrospective use of imaging data acquired as part of the clinical evaluations for diagnosis of possible Chiari I malformation. Inclusion criteria for classification as a patient with Chiari I were signs and symptoms of a Chiari I malformation and clinical improvement following craniocervical decompression. 2D FIESTA studies were performed in conjunction with routine MR brain imaging in 11 patients with a Chiari I malformation (4 men and 7 women, 29-50 years of age) and in 6 patients without tonsil ectopia (20-50 years of age). Four of the 11 patients with Chiari I had syringohydromyelia. The 6 subjects without tonsil ectopia, though referred because of its possibility, had no clinical symptoms or signs of a Chiari malformation.

Received November 20, 2008; accepted after revision December 16.

From Advanced Diagnostic Imaging (J.C.), Saginaw, Mich; and Department of Radiology (V.H.), University of Wisconsin School of Medicine and Public Health, Madison, Wis.

Please address correspondence to Victor Haughton, MD, University of Wisconsin Hospitals and Clinics, Department of Radiology, E3/311 Clinical Science Center, 600 Highland Ave, Madison, WI 53792; e-mail: vmhaughton@wisc.edu

DOI 10.3174/ajnr.A1507

\section{D FIESTA Imaging}

Localizer images, T1- and T2-weighted cervical spine images, and sagittal 2D FIESTA images were acquired as part of the current clinical protocol for imaging in patients with a possible Chiari I malformation. The T1- and T2-weighted images were inspected for evidence of tonsil ectopia and for the presence of a syrinx.

For 2D FIESTA, 3 contiguous sagittal sections centered on the foramen magnum were acquired with peripheral electrocardiography gating; 20 frames per cycle; TE/TR, 1.2/6.2 ms; flip angle, $45^{\circ}$; $384 \times$ 256 matrix; 20-cm FOV, and an in-plane resolution of $0.5 \times 0.78 \mathrm{~mm}$.

The FIESTA study was reviewed as a cine loop. The inferior margin of the tonsil was identified in each phase of the cardiac cycle by 1 investigator, and the motion of that margin between the most cephalad and most caudad positions was measured with distance-measuring software in the PACS system, referred to as manual measurements. Motion of the tonsils between images was also measured by a software program based on pixel shifting. ${ }^{8}$ For this measurement, 1 investigator placed a region of interest of 60 pixels in diameter on the initial image to encompass the tonsils, inferior cerebellum, and brain stem and exclude the cranium and clivus. The program then calculated the amount of translation in a superior-inferior direction in the consecutive images to align the selected tissues. The translation in the superior-inferior direction measured with respect to the first image was plotted as a function of trigger delay. Tonsil motion was calculated as the difference between the most superior and inferior displacements of the tonsils.

The displacements of the tonsils measured by the automated program were averaged for patients with Chiari I and controls and compared; mean tonsil displacement was calculated for patients with and without syringohydromyelia and compared. The measurements made with the pixel-shift program were compared with manual measurements.

\section{Results}

\section{D FIESTA Imaging}

In subjects with a normal cerebellar tonsil position (controls) and in patients with Chiari I, small displacements of the tonsils were evident on inspection of the 2D FIESTA acquisitions that were not conspicuously different between patients and controls or between subjects with and without syringomyelia.

Plots of tonsil displacement from the initial image showed a biphasic change in tonsil position during the cardiac cycle (Fig 1). Tonsil displacement calculated from the pixel-shift 

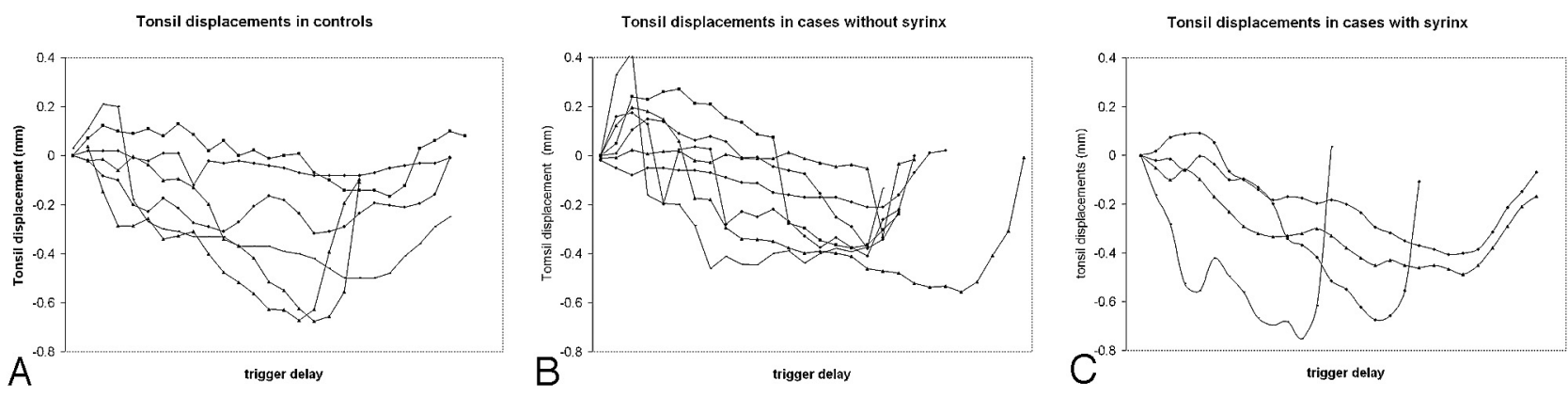

Fig 1. Plots of tonsil displacement with respect to trigger delay for the controls $(A)$, patients with Chiari I without syrinx $(B)$, and patients with Chiari I with syrinx (C).

Tonsil displacements during the cardiac cycle in controls and patients with Chiari I malformation with and without syringomyelia

\begin{tabular}{|c|c|c|c|c|}
\hline & No. & $\begin{array}{c}\text { Tonsil } \\
\text { Displacement } \\
(\mathrm{mm})\end{array}$ & $\mathrm{SD}(\mathrm{mm})$ & Range (mm) \\
\hline Controls & 6 & 0.43 & 0.06 & $0.12-0.71$ \\
\hline Patients with Chiari I & 11 & 0.57 & 0.04 & $0.23-0.78$ \\
\hline Without syringomyelia & 7 & 0.50 & 0.05 & $0.23-0.71$ \\
\hline With syringomyelia & 4 & 0.61 & 0.03 & $0.45-0.78$ \\
\hline
\end{tabular}

program averaged $0.43 \mathrm{~mm}( \pm 0.06 \mathrm{~mm})$ for the control subjects (Table). For patients with Chiari I, the tonsil motion averaged $0.57 \mathrm{~mm}( \pm 0.04 \mathrm{~mm})$ or larger $(33 \%)$. For patients with Chiari I without syringohydromyelia, tonsil motion averaged $0.5 \mathrm{~mm}( \pm 0.05 \mathrm{~mm})$; and for patients with Chiari I with syringohydromyelia, it averaged $0.61 \mathrm{~mm}( \pm 0.03 \mathrm{~mm})$ or larger $(22 \%)$.

\section{Discussion}

The study shows that successive cephalad and caudad cerebellar tonsil motion is detectable on cine MR imaging during the cardiac cycle. Tonsil motion differed by submillimeter amounts between patients and controls. The study suggested a trend toward greater motion in patients than in controls and a weak trend toward greater displacement in patients with than without syringomyelia.

The results of this study agree with those of Levy, ${ }^{2}$ who found little movement of the tonsils and spinal cord during the cardiac cycle on PC MR images. Our findings are concordant with the theory that brain movement transfers directly to the spinal cord and tonsils. ${ }^{4,5}$ Our results differ from those of Oldfield et al, ${ }^{1}$ whose sonography studies after $\mathrm{C} 1$ laminectomy in patients with Chiari I demonstrated large displacements of the tonsils in those patients. Whether $\mathrm{C} 1$ laminectomy or general anesthesia increased tonsil motion in their subjects has not been determined.

The automated pixel-analysis program has a high degree of precision and accuracy for measuring rotations of vertebrae. ${ }^{8}$ For measuring soft-tissue displacements, the method has less accuracy and precision. Because motion is averaged over multiple voxels, the method may achieve greater precision than the in-plane dimensions of the voxel, that is, $0.5 \times 0.78 \mathrm{~mm}$. Because the differences between groups were small relative to the pixel size, $P$ values were not calculated. Manual measurements concur with the automated measurements, though they have the drawbacks of reader bias and limited accuracy.

A limitation of the study is the small study population. The small differences we noted between groups might be significant if the statistical power of the study was greater. However, inspection of the 2D FIESTA images showed similar patterns of tonsil motion in patients and in controls. Sagittal PC MR magnitude images acquired regularly in MR imaging evaluation of the Chiari I malformation also showed submillimeter tonsil movement but, because of the poor contrast between tissue and CSF, did not permit ready measurement.

\section{Conclusions}

The cerebellar tonsils move biphasically less than a millimeter during the cardiac cycle in both control subjects and patients with Chiari I malformations. Preoperative 2D FIESTA imaging might be a useful adjunct to intraoperative sonography to determine the role of tonsil motion in the pathogenesis of syringohydromyelia.

\section{References}

1. Oldfield EH, Muraszko K, Shawker TH, et al. Pathophysiology of syringomyelia associated with Chiari I malformation of the cerebellar tonsils. J Neurosurg 1994;80:3-15

2. Levy LM. Toward an understanding of syringomyelia: MR imaging of CSF flow and neuraxis motion. AJNR Am J Neuroradiol 2000;21:45-46

3. Greitz D, Franck A, Nordell B. On the pulsatile nature of intracranial and spinal CSF-circulation demonstrated by MR imaging. Acta Radiol 1993; 34:321-28

4. Feinberg DA, Mark AS. Human brain motion and cerebrospinal fluid circulation demonstrated with MR velocity imaging. Radiology 1987;163:793-99

5. Enzmann DR, Pelc NJ. Brain motion: measurement with phase-contrast MR imaging. Radiology 1992;185:653-60

6. Maier SE, Hardy CJ, Jolesz FA. Brain and cerebrospinal fluid motion: real-time quantification with M-mode MR imaging. Radiology 1994;193: 477-83

7. Poncelet BP, Wedeen VJ, Weisskoff RM, et al Brain parenchyma motion: measurement with cine echo-planar MR imaging. Radiology. 1992;185: 645-51

8. Rogers B, Wiese S, Blankenbaker D, et al. Accuracy of an automated method to measure rotations of vertebrae from computerized tomography data. Spine 2005;30:694-96 